\title{
Aerodynamic CFD Based Optimization of Police Car Using Bezier Curves
}

\author{
Ali Reza Taherkhani and Carl Gilkeson PhD \\ University of Leeds \\ Philip Gaskell PhD \\ Durham University \\ Rob Hewson PhD \\ Imperial College \\ Vassili Toropov $\mathrm{PhD}$ and Amin Rezaienia $\mathrm{PhD}$ \\ Queen Mary University of London \\ Harvey Thompson \\ University of Leeds
}

\begin{abstract}
This paper investigates the optimization of the aerodynamic design of a police car, BMW 5-series which is popular police force across the UK. A Bezier curve fitting approach is proposed as a tool to improve the existing design of the warning light cluster in order to reduce drag. A formal optimization technique based on Computational Fluid Dynamics (CFD) and moving least squares (MLS) is used to determine the control points for the approximated curve to cover the light-bar and streamline the shape of the roof. The results clearly show that improving the aerodynamic design of the roofs will offer an important opportunity for reducing the fuel consumption and emissions for police vehicles. The optimized police car has $30 \%$ less drag than the non-optimized counter-part.
\end{abstract}

CITATION: Taherkhani, A., Gilkeson PhD, C., Gaskell PhD, P., Hewson PhD, R. et al., "Aerodynamic CFD Based Optimization of Police Car Using Bezier Curves," SAE Int. J. Mater. Manf. 10(2):2017, doi:10.4271/2017-01-9450.

\section{INTRODUCTION}

Over the past several decades, a significant amount of effort has been invested to reduce the aerodynamic drag of heavy goods vehicles since this directly influences the fuel consumption and dynamic performance, a reduction in total aerodynamic drag leads into a significant improvement in the fuel economy. In recent years the number of studies which have applied Computational Fluid Dynamics (CFD) and optimization methods to improve the aerodynamic design of road vehicles has increased significantly and is recognized as providing a powerful and efficient means of generating improved aerodynamic performance, particularly when detailed experimentation in wind tunnels is not achievable, see e.g. $[\underline{1}, \underline{2}, \underline{3}, \underline{4}, \underline{5}, \underline{6}]$. The focus of recent studies has been on the drag reduction of Heavy Goods Vehicles, see e.g. $[\underline{1}, \underline{4}, \underline{5}, \underline{6}, \underline{7}]$. Currently there have been relatively few studies on the aerodynamic improvement of light trucks and cars $[\underline{8}, \underline{9}]$, while a small number still have reported reduced aerodynamic drag using automated design optimization techniques $[\underline{2}, \underline{8}, \underline{10}]$.
The present work focuses on the aerodynamic design optimization of a modified BMW 5-series police car, such as those commonly by our police sources and whose aerodynamic optimization has not been considered previously in the literature. It complements and extends a recent study into aerodynamic optimization of ambulances $[\underline{10}, \underline{11}]$.

One of the most important ingredients in numerical optimization is the choice of design variables and the parameterization methodology employed $[12, \underline{13}]$. In order to reduce the number of parameters needed to describe the shape of the roof, several mathematical shape-capturing methods have been proposed in the literature [14]. In the present study, a third order Bezier curve is used to describe the geometry. The advantage of this choice is that, with only two design variables, a wide range of roof dimensions can be assessed [12]. Past methods often involve complex parameterization schemes which use arcs, circles and polynomials for geometry creation. Many of these methods require large data sets to describe the profiles. In addition, they do not provide simple, intuitive parameters for the designers to control [15]. Bezier curves are used to fit an existing data set while maintaining tangency and curvature conditions. Bezier curves have 
since become a popular method for creating parametric curves and they have wide applications including turbine blade design [16], wind turbine airfoil design $[\underline{12}, \underline{16}]$, face recognition [17] .

Computational Fluid Dynamics (CFD) is used within a multiobjective optimization framework to minimize the drag force on a police car, based on the BMW 5-series design. Design parameterization of a three-dimensional real car model is carried out to enable optimization for a wide range of roof configurations. Optimal Latin Hyper-cubes for surrogate model building and model validation points are constructed using a permutation genetic algorithm and design points are evaluated using CFD. Surrogate models are built using a Moving Least Squares approach.

Optimizations of the roof configuration, was undertaken using a genetic algorithm with responses calculated from the surrogates. This approach results in a set of optimized designs, from which appropriate roof designs with lower drag can be obtained. The results presented show significant potential for aerodynamic drag and emissions reductions of police cars.

The choice of design variables in the geometry parameterization is one of the most important part of the optimization process [12]. Previous studies have often used complex parameterization schemes which use arcs, circles and polynomials for geometry creation [15]. Many of these methods require large data sets to describe the profiles. In addition, they do not provide simple, intuitive parameters for designers to control [15]. Therefore there is a need for a method flexible enough to fill the design space. Bezier curves are one such option since they can be controlled by any desired control points.

The equation of the n-order Bezier curve is:

$$
p(t)=\sum_{i=0}^{n} B_{i}^{n}(t) \cdot P_{i}
$$

where $P_{i}$ represents the set of $\mathrm{n}+1$ control points, $t$ is a parameter that varies between 0 to 1 , and $B_{i}^{n}(t)$ is the blending function:

$$
\begin{aligned}
B_{i}^{n}(t) & =\left(\begin{array}{c}
n \\
i
\end{array}\right) \cdot(1-t)^{n-i} \cdot t^{i}, \\
i & =0,1, \ldots \ldots \ldots ., n,
\end{aligned}
$$

In the blending function formula $\left(\begin{array}{c}n \\ i\end{array}\right)$ is the binomial coefficient given by $\frac{n !}{i !(n-i) !}$, where $\mathrm{n}$ defines the Bezier curve's degree of freedom.

From figures 1 and equations 1 and 2 , it can be seen that the blending functions act as a link between the control points and the points on the Bezier curve. In each case the first and last control points lie on the actual curve while the other control points form a control envelope for the curve. Often a single Bezier curve is insufficient for describing a complex profile and several of them are joined together. In our case only one Bezier curve with four control points is sufficient as it would give us wide range of roof dimensions in our design space.
Figure 1 shows Bezier curves with different number of control points:

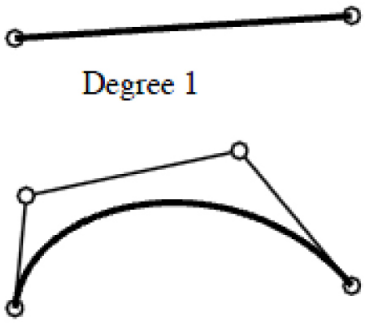

Degree 3

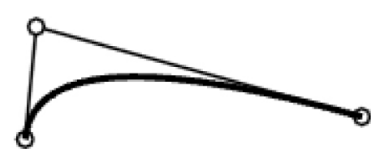

Degree 2

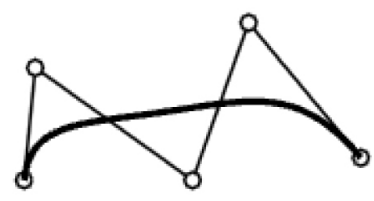

Degree 4
Figure 1. Bezier curves of various control point and degree of freedom

\section{CFD METHODOLOGY}

\section{CFD Modeling}

The geometry of the vehicle is shown in figure 2 which is based on a simplified model of the BMW 5-series used in the UK police fleet with the dimensions below.

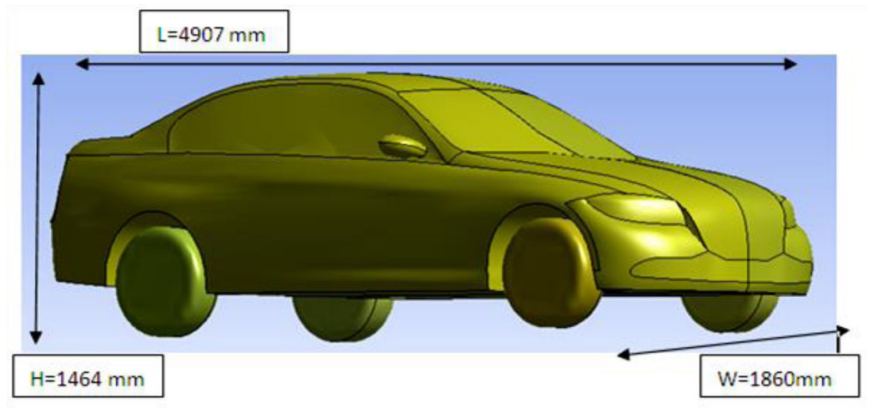

Figure 2. Simplified model of BMW 5series

Only symmetrical, zero yaw angle air flow cases are considered, enabling the flow domain to be reduced by half in order to reduce the computational costs. The semi-cylindrical domain has the following dimensions (See figure 3):

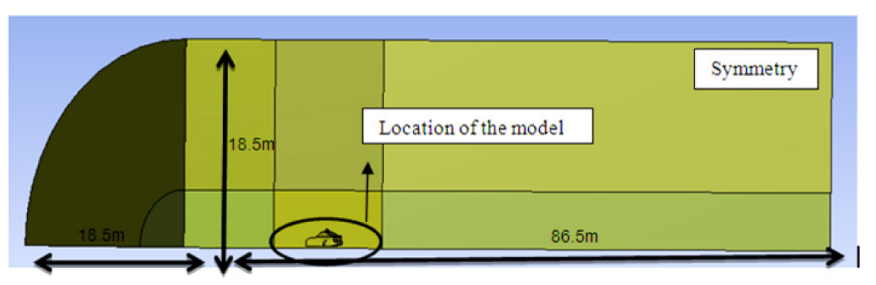

Figure 3. Wind tunnel domain with its 3D dimensions

These dimensions are large enough to minimize any flow blockage effects and to adequately capture turbulent flow behind the vehicle [11], the domain was decomposed into 6 zones to allow suitable mesh control. The volume immediately surrounding the vehicle was discretised by tetrahedral elements, in order to accurately define the complexity of the vehicle geometry, with mesh refinement increasing towards the vehicle surface. Other volumes were meshed using structured elements, see figure 4. 


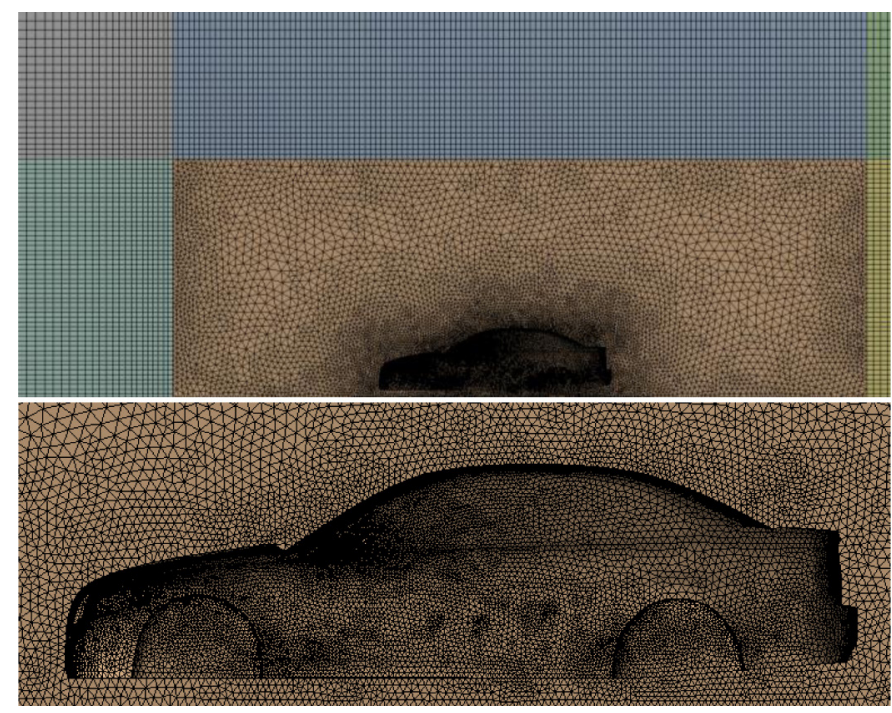

Figure 4. Illustration of the mesh type and size, wind tunnel (top, unstructured around the vehicle and structured further away from the vehicle to reduce the number of elements) and around the vehicle (bottom, smaller elements close to the surface of the vehicle and specially around small parts like mirrors)

In practice the mesh density used throughout the optimization is a compromise between the computation time and simulation accuracy. In this example the generated mesh contains around 9.7 million elements with a cell size of 10 millimetres on the vehicle surface, which correspond to a $y^{+}$value of 75 which is within the range of 30-500 in order to reduce the affects of the turbulence boundary layer [18]. Such mesh densities have been shown to provide good agreement with corresponding wind tunnel data for emergency response vehicles [11].

\section{Boundary Condition}

At the inlet flow boundary, located upstream of the vehicle, a uniform stream-wise velocity of $26.8 \mathrm{~m} / \mathrm{s}(60 \mathrm{mph})$ was prescribed, while at the outlet an atmospheric pressure condition was specified.

Table 1. Boundary conditions applied for the generic ambulance model

\begin{tabular}{|l|l|l|}
\hline Boundary & Boundary type & Parameters \\
\hline Inlet & Velocity Inlet & $26.8 \mathrm{~m} / \mathrm{s}$ \\
\hline Outlet & Pressure Outlet & $0 \mathrm{~Pa}$ \\
\hline Road & Moving wall & $26.8 \mathrm{~m} / \mathrm{s}$ \\
\hline Tyres & Rotating wall & $70.5 \mathrm{rpm}$ \\
\hline Vehicle surface & Stationary wall & No slip \\
\hline Domain top & Stationary wall & Zero shear stress \\
\hline
\end{tabular}

The same velocity was set for the moving ground. At the wheels, the velocity boundary condition was set to provide a rotation with the circumferential speed being equal to that of the moving ground. On the surface of the vehicle, a no-slip wall boundary condition was specified. A symmetry boundary condition was applied at the vertical edge of the flow domain. The boundary conditions are summarized in Table 1.

\section{Mesh Independence}

A mesh dependency study was performed to assess the effect of mesh size on CFD predictions for the police car. Different mesh sizes with two different turbulence models were assessed. As well as the mesh on the surface of the vehicle, the mesh for the whole wind tunnel was iteratively changed.

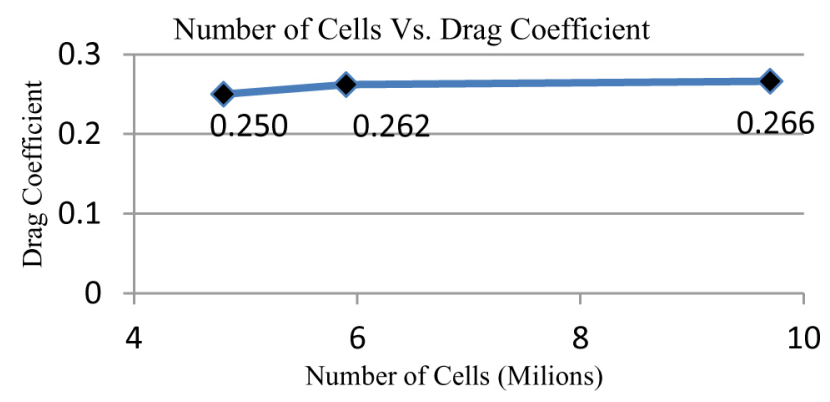

Figure 5. Graph of mesh dependency for Standard k - $\varepsilon$

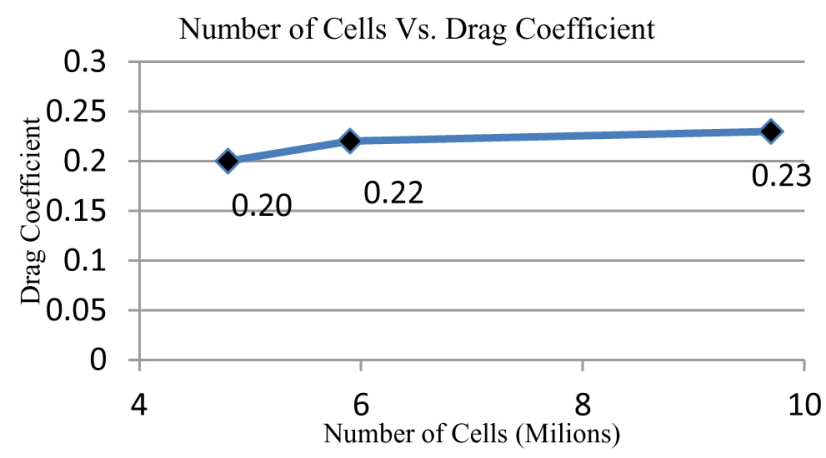

Figure 6. Graph of mesh dependency for Realizable k - $\varepsilon$

This study indicates the independency of the results from the mesh density and as the mesh density increases from 5 to 10 million elements, the drag coefficient ranges from 0.250 to 0.266 for Standard $k-\varepsilon$ and 0.20 to 0.23 for Realizable $k-\varepsilon .5$ million cells, which relates to cell size of $10 \mathrm{~mm}$ on the surface of the vehicle (minimum cell size), were used to mesh the model based on these results.

\section{Turbulence Model Validation}

Several previous studies have shown that the family of two equation $k-\varepsilon$ models can be used to successfully predict aerodynamic drag on vehicles with dimensions similar to ambulance geometries, see e.g. $[\underline{8}, \underline{9}, \underline{19}, \underline{20}]$. The predictions from the two such models were compared with experimental data for a real model of BMW 5-series which has a drag coefficient of 0.27 [21] . The following turbulence models (see table 2) were used to simulate the drag coefficient and find the most suitable with the drag coefficient closest to experiment: Table 2 presents the results obtained from the simulations with the element size of 10 millimeters:

Table 2. Drag coefficients for different turbulent model

\begin{tabular}{|c|c|}
\hline Turbulence Model & Drag Coefficient \\
\hline$k-\varepsilon$ standard & 0.266 \\
\hline$k-\varepsilon$ Realizable & 0.230 \\
\hline Experimental & 0.270 \\
\hline
\end{tabular}


The prediction with the $k-\varepsilon$ standard model agrees with the experimental value to 2 decimal places and has a discrepancy of $1.5 \%$.

Along with the previous literature, the following result confirms that the standard $k-\varepsilon$ model is the most suitable model to capture the flow behaviour around the vehicle.

\section{Impact of Light Bars}

Table 3 shows that adding a typical light bar (Conversion A, Figure 5) to the benchmark model increases the drag force by $34 \%$.

Table 3. Drag forces comparison for car with and without light bar

\begin{tabular}{|l|l|l|}
\hline Model & Drag force (N) & Drag difference (\%) \\
\hline Base & 172.8 & $\ldots \ldots \ldots .$. \\
\hline Converted with light-bar & 232.1 & +34 \\
\hline
\end{tabular}

The light bar which is located on the middle of the roof is an obstacle for the oncoming flow and it forces the flow to change its direction abruptly, causing separation and an area of low pressure as it can be seen in figure 7 (dark blue area). The larger areas of separation behind the light bar and at the back of the vehicle leads to greater aerodynamic drag, see figure 7.

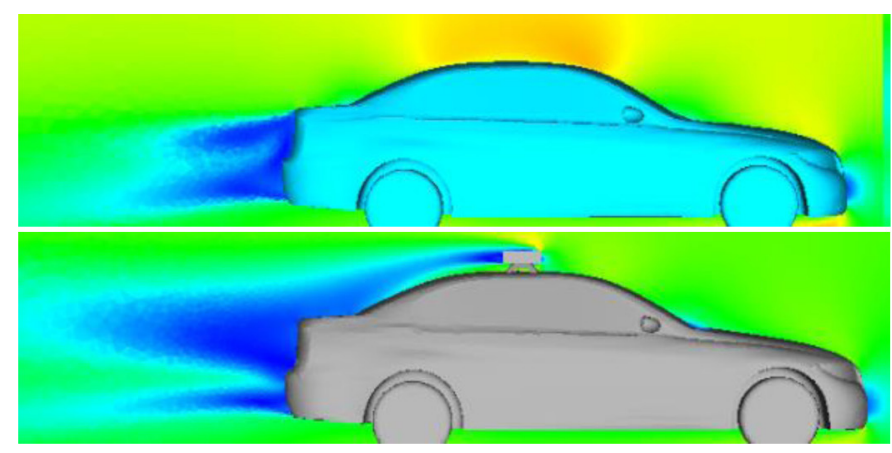

Figure 7. velocity contour for base car (top) and converted one with light bar (bottom)

\section{INITIAL PARAMETER STUDY}

In order to investigate the effects of the different parameters on the drag force produced by the light-bar, the light bar was represented parametrically in terms of the parameters shown in figure 8 and the length was kept constant.

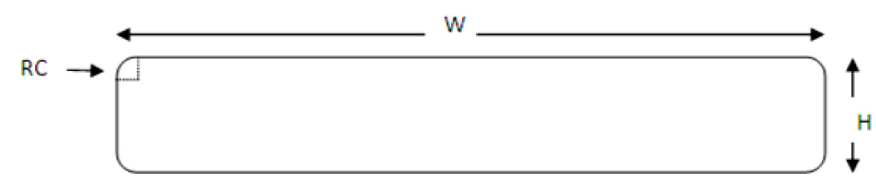

Figurer 8. light-bar with the location of its parameters

- $\quad$ Radius of curvature, rc ( 0 to $50 \mathrm{~mm}$ )

- Height, H ( 50 to $100 \mathrm{~mm}$ )

- Width, W (200 to $310 \mathrm{~mm}$ )

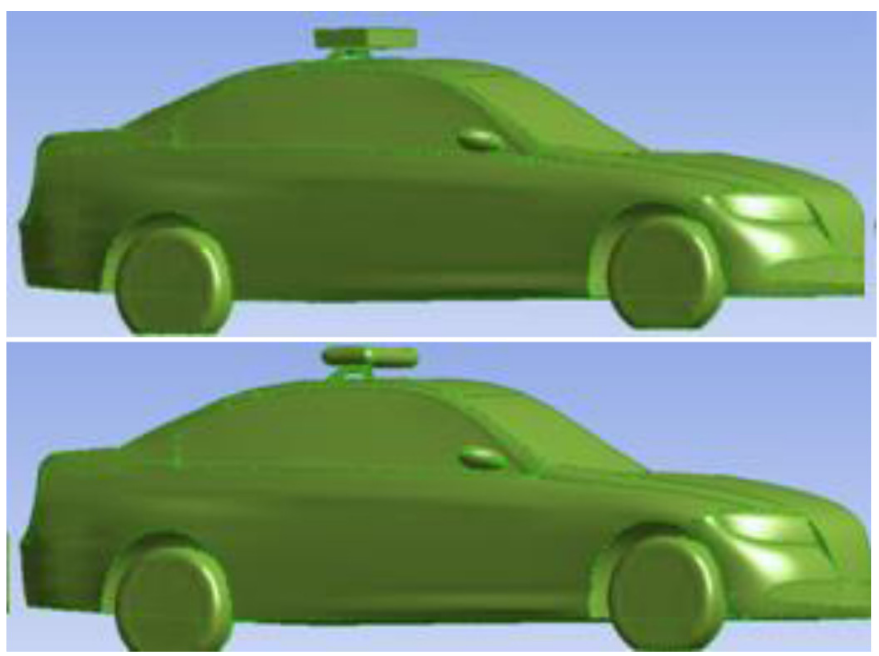

Figure 9. light bar without (top) and with(bottom) rounded edge

Since the simulation is expensive, in an initial study only corner point conditions were simulated to see how influential they are with respect to drag force production:

Table 4. Results of parametric study of the light-bar

\begin{tabular}{|c|c|c|c|}
\hline Radius of curvature & \multicolumn{1}{|l|}{ Height } & \multicolumn{1}{l|}{ width } & Drag force (N) \\
\hline 0 & 50 & 200 & 195 \\
\hline 0 & 50 & 310 & 194 \\
\hline 0 & 100 & 200 & 241 \\
\hline 0 & 100 & 310 & 232 \\
\hline 50 & 50 & 200 & 181 \\
\hline 50 & 50 & 310 & 181 \\
\hline 50 & 100 & 200 & 196 \\
\hline 50 & 100 & 310 & 199 \\
\hline
\end{tabular}

From the above results, it can be seen that as the radius of curvature changes from 0 to the maximum of $50 \mathrm{~mm}$ while other parameters are kept constant, the drag force decreases considerably from between $7 \%$ to $22 \%$ for different heights and widths. The drag force also increases as the height increases. Note that the width of the light bar does not influence the drag force and the minimum drag force corresponds to the maximum radius of curvature and minimum height. Therefore the frontal area plays an important role in drag production while the light bar's width is much less influential.

Another study was conducted into the effect of the height of the light-bar on the drag force it produces, see fig 8. The light bar was placed directly on the roof without any stands to see the effect the stand has on the drag force. The drag force reduces from $232 \mathrm{~N}$ to $225 \mathrm{~N}$ when the light bar is placed directly on the roof as shown in figure 10.

This modification is permitted by the regulations governing lighting for real vehicle [22] , so that optimization was performed for a model with zero height light-bar on the roof, see figure 10. 


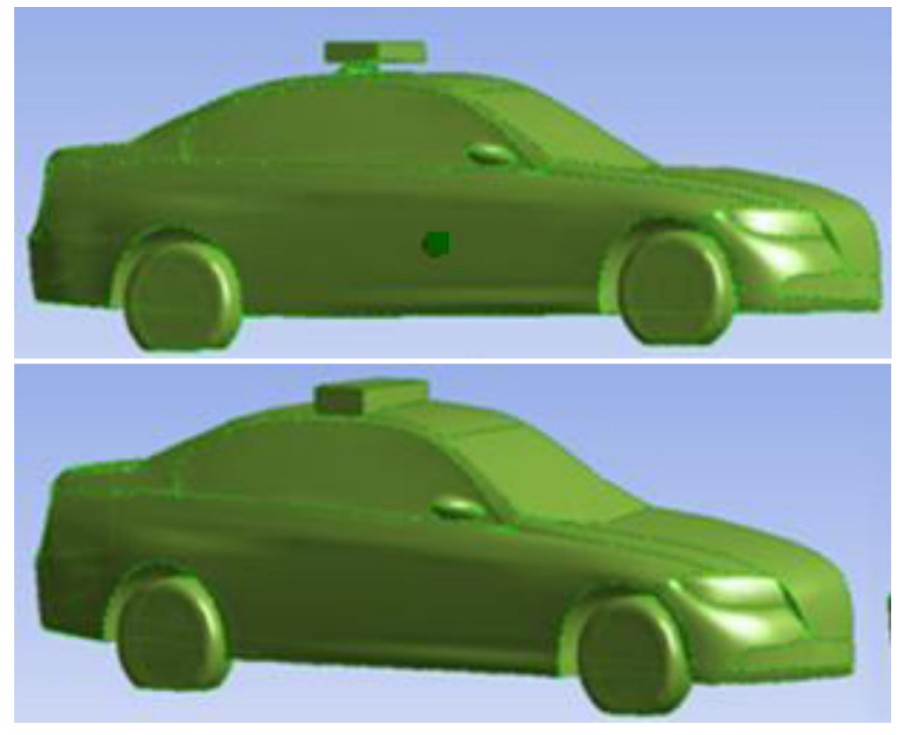

Figure 10. light bar with 48 (top) and 0 (bottom) mm height

These parametric studies show the sensitivity of aerodynamic drag to the light bar geometry. The next step is to carry out a formal design optimization of the light bar shape based on Bezier curves.

\section{MODEL PARAMETERIZATION}

The key issue prior to carrying out design optimization on the vehicle's roof is to choose an efficient parameterization scheme for modifying its shape. As three dimensional CFD simulations are computationally expensive, it is desirable that the parameterization should be based on a small set of design variables since adding design variables increases optimization time dramatically. In the present work the parameterization is based on the tools provided by the commercial CFD software ANSYS Work-Bench [를 which was used for creating the geometry, modelling and mesh generation.

A Bezier curve has been chosen in order to represent the potential (optimal) configuration of the roof of the BMW 5series Police car conversion with an embedded light bar. A cubic Bezier curve which has 4 control points was used and has been described by the following expression:

$$
\begin{aligned}
B(t)=(1-t)^{3} P_{0} & +3(1-t)^{2} t P_{1}+\ldots \\
& +3(1-t) t^{2} P_{2}+t^{3} P_{3}, t \in[0,1]
\end{aligned}
$$

where $t$ is a parameter that varies between 0 to 1 and p represents the control points. The Bezier curve profile was defined using 100 equally distributed points.

\section{Definition of Design Variables}

Two design variables were used for shape optimization of the vehicle roof. These are linked to the length (figure 11, top) and sharpness (figure 11, bottom) of the curve and the height of the light bar remains unchanged during the optimization.

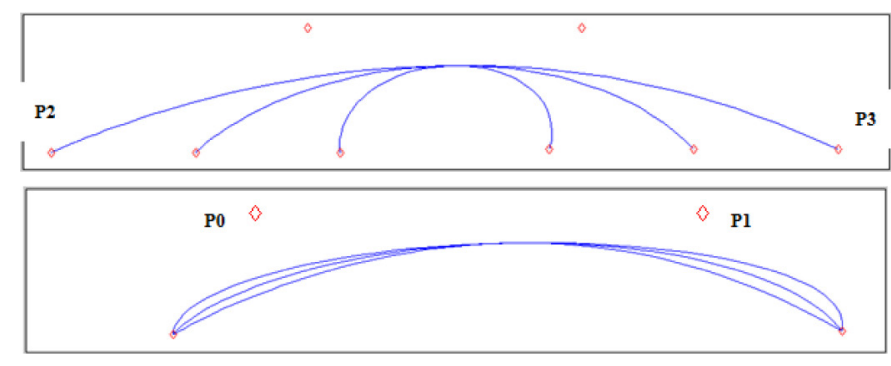

Figure 11. Design parameter 1, sharpness (top) and design parameter 2, length (bottom)

The distance between $P_{0}$ and $P_{1}$ determines the sharpness of the curve and distance between $P_{2}$ and $P_{3}$ determines the length of the curve.

\section{OPTIMIZATION METHOD}

The objective function is to minimize the aerodynamic drag force $\mathrm{D}$ (in Newtons) given by $D=\frac{\rho A V^{2} C_{D}}{2}$, where $\rho$ is the air density, A the frontal area, $\mathrm{V}$ free-stream velocity and $C_{D}$ is the drag coefficient.

Since D is proportional to the product of the vehicle frontal area and the drag coefficient, this objective function, for a given density and velocity, represents a compromise between the size and aerodynamic performance of the vehicle.

\section{Optimization Strategy}

The goal of optimization is to find a set of design variables $\boldsymbol{x}$ which optimizes (minimizes or maximizes) an objective or cost function $f(\boldsymbol{x})$ [24] subject to certain, defined constraints.

Numerical optimization techniques can be broadly classified as either deterministic [25] or stochastic. The nonlinear nature of flow phenomena inside enclosed spaces, such as a wind tunnel, leads to discontinuous outputs being generated which in turn causes problems for deterministic methods [26]. Since these methods are based on mathematical procedures, in most cases they are generally prone to find the local extreme and the convergence speed and the value of the final results are strongly dependent on the initial values chosen [27]. In contrast, stochastic methods, also referred to as global methods are better suited to wind tunnel and indoor applications. One of the most popular in this category and a widely accepted global optimization technique is the Genetic Algorithm (GA) method [27]. Inspired from Darwin's theory of natural selection, this method has demonstrated its capability to handle discontinuous variables and also noisy objective functions [료]. In addition it can find near optimal solution using less computing time compared to other methods such as mixed-integer programming [29].

Furthermore, GA being a stochastic method has a better chance to explore the entire design space and reach global optimum. Hence GA is selected as the optimization engine in this study. Multi-objective optimization methods that are based on evolutionary algorithms, especially multi-objective GAs, require hundreds or sometimes 
thousands of evaluations of the objective functions to search for the optimal solutions [30]. In aerodynamic applications, where evaluation of the objective function comes from computationally expensive and time consuming CFD simulations, the optimization process could therefore take a prohibitively long time to achieve its goal. Hence, in order to save computational time associated with a GA, a Response Surface Method (RSM) method is used to mimic the behavior of the system response with respect to the change in design variables. The RSM models which are constructed from high-fidelity simulations provide fast approximations of the objective and constraint functions at new design points, thereby saving computational time and making optimization studies using GA feasible [31] .

A surrogate modelling approach is adopted for the optimization study. Design of experiments (DoE) is carried out using an Optimal Latin Hypercube containing build and validation points. This is achieved via a permutation genetic algorithm applied to the multi-objective problem of optimizing the uniformity of model building points, model validation points, and combined DoEs. The optimality criterion for each DoE is defined by the Audze-Eglais method [32] with the objective function defined by:

$$
\begin{gathered}
U=\sum_{i=1}^{P} \sum_{j=i+1}^{P} \frac{1}{L_{i j}} \\
F=W_{b} U_{b}+W_{v} U_{v}+W_{m} U_{m}
\end{gathered}
$$

where $U$ is a pseudo-potential energy of DoE points, $L_{i j}$ is the distance between points $i$ and $j$ where $i \neq j, F$, the objective function, is to be minimized, $W$ are weighting factors, and $b, v, m$ denote model building, model validation and combined DoEs respectively. Surrogate models were built using a Moving-Least-Squares (MLS) method where the weighting of points in the regression coefficients calculation is determined using a Gaussian decay:

$$
w_{i}=\exp \left(-\theta r_{i}^{2}\right)
$$

where $w$ is the weighting of the DoE build point $i, r_{i}$ is the normalized distance from the current point to model building point $i$, and $\theta$ is a closeness-of-fit parameter.

This parameter is optimized to minimize the $R^{2}$ value for the obtained surrogate model, as calculated on the validation DoE. The surrogate is then rebuilt using the combined building and validation DoEs. Global optimization is performed using a genetic algorithm (GA) with responses calculated using the surrogate models. The optimized design variables from the GA are then fine-tuned using a Sequential Quadratic Programming method to ensure an optimum has been reached. Figure 12 shows the optimization framework.

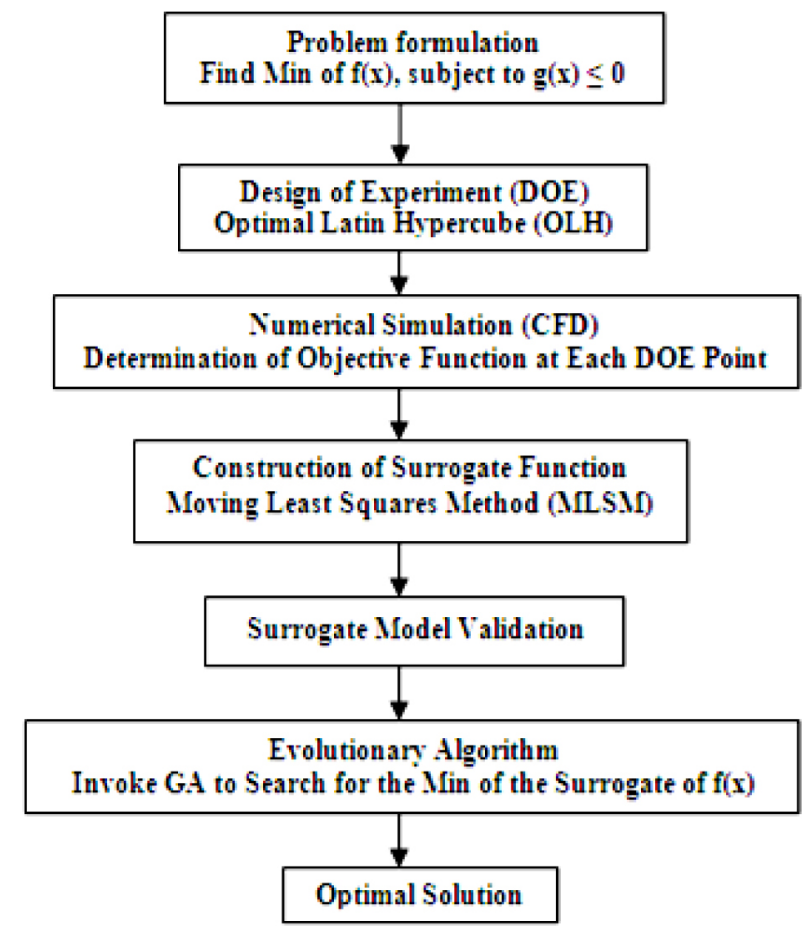

Figure 12. Optimization Framework [푸]

\section{OPTIMIZATION RESULT}

A 25 point optimal Latin hypercube DoE is constructed using the approach described earlier. Of the 25 points, 15 are building points and 10 are validation points. The distribution of points in the design variable space is shown in Figure 13.

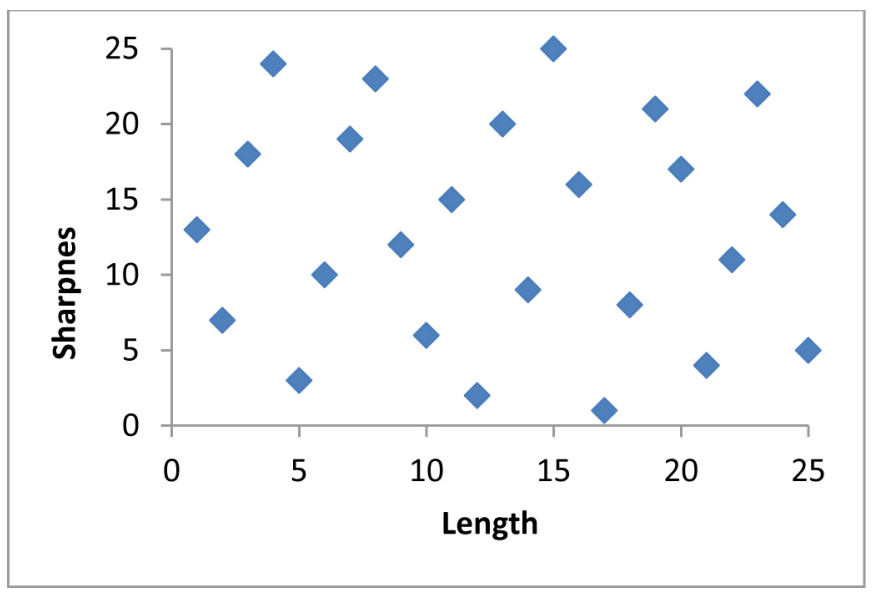

Figure 13. Distribution of normalized design points in design variable space

Values of the standard deviation of minimum distances $\sigma_{b}, \sigma_{v}$ and $\sigma_{m}$ for building, validation and merged DOEs are found to be $\sigma_{b}=0.56$, $\sigma_{v}=1.26$ and $\sigma_{m}=0.54$, respectively.

High fidelity analysis is performed using the steady-state solution to the governing Navier-Stokes equations for 3D flow using the commercial CFD software FLUENT v14.0 (ANSYS 2012) and the optimization was carried out using Hyper-Study v11 software (Altair 
Engineering, Hyper-Works 2012). Second order upwind discretization schemes are used for all flow variables and solutions are computed using the SIMPLE algorithm [34].

The Drag Force for each design point is extracted from the CFD data. MLS approximations of the response is then constructed using a second order base polynomial and the 15 model building points. The closeness of fit parameter is optimized using the 10 model validation points. MLS surfaces gave equally good agreement with building and combined DOEs ( $\mathrm{R}^{2}$ values of 0.9883 and 0.904 for building and combined, respectively). However, there was a slight difference in agreement with the validation points $\left(\mathrm{R}^{2}\right.$ values of 0.811$)$. The surrogate function was then used in conjunction with GA to find the minima, using a second order base polynomial and the 15 model building points.

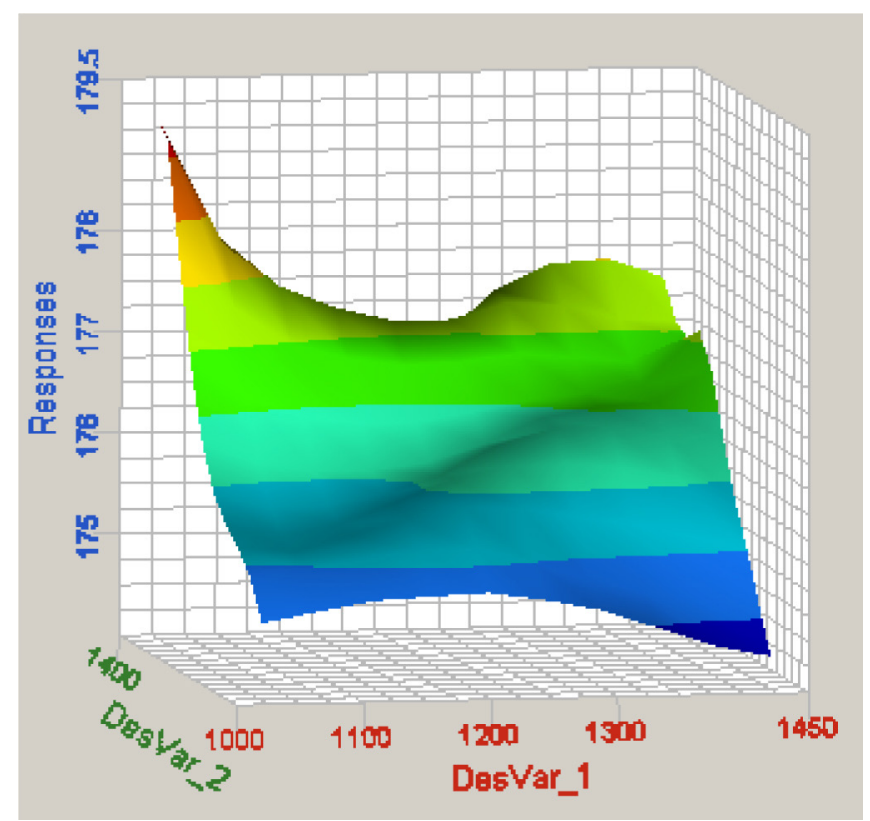

Figure 14. Response surface of the drag coefficient as a function of both design variables

The optimization problem was formulated in order to minimize the objective function, drag. A Genetic algorithm (GA) was used to find a global minimum with fitness evaluations carried out by the surrogate models. The GA produced a design which, as predicted by the surrogate model, would reduce drag force. CFD studies were made of the optimized designs They showed good agreement with the surrogate models with a $\sigma_{D}=174.36 \mathrm{~N}$ and $\sigma_{D}=173.43 \mathrm{~N}$ which are within $0.5 \%$ of the surrogates' predictions. A summary is given in Table 5.

Table 5. Comparison of the responses obtained from the optimization and CFD

\begin{tabular}{|l|c|}
\hline \multicolumn{1}{|c|}{ Model } & Responses \\
\hline Optimized design after GA & $174.36(\mathrm{~N})$ \\
\hline CFD validation from optimum & $173.43(\mathrm{~N})$ \\
\hline Error (Percentage) & $0.5 \%$ \\
\hline
\end{tabular}

The dark blue area in figure 14 corresponds to the minimum drag which indicates the minimum drag mainly depends on the sharpness of the Bezier curve and the length of the curve is less influential on the variation of the drag force. Therefore the minimum drag is when the length and the sharpness of the curve are at its maximum. Since the optimum is the corner point, there is no need to re-optimize with the new data point and figure 15 shows the CFD model of the optimized design. The results of the optimization are summarized in $\underline{\text { table } 6}$, which shows the drag calculation for the generic police car, its conversion with light-bars and the optimized design.

Table 6. Drag comparison for the original and optimized design

\begin{tabular}{|l|l|l|}
\hline Designs & Drag force $(\mathrm{N})$ & Drag Difference (\%) \\
\hline Base Car & 172.8 & \\
\hline Conversion A ( 48 mm height) & 232 & +34.2 \\
\hline Conversion B (0 mm height) & 225 & +30.2 \\
\hline Optimized design & 173.43 & +1 \\
\hline
\end{tabular}

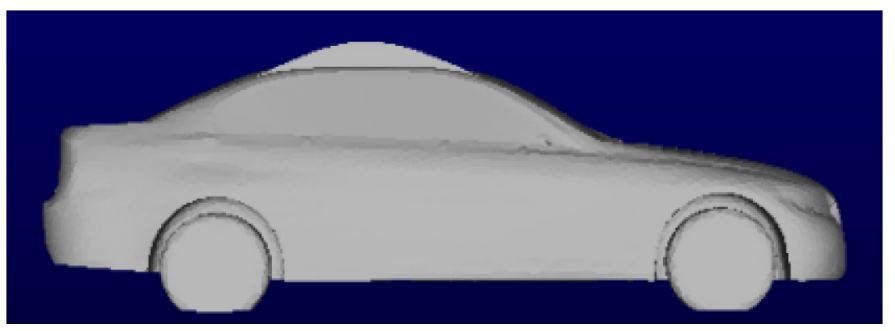

Figure 15. Optimum configuration of the car with light bar

$\underline{\text { Figure } 16}$ and 17 show the contours and streamlines of the velocity for the original, conversion and the Bezier curve based design. Blue colour in the figures indicates the area of low pressure which is the main cause of drag production. For the base car, this area is only at the rear of the vehicle and is as a result of separation and circulation of the flow. For the car with a light bar, the blue area extends to the roof and just after the light bar, since the light bar acts as an obstacle and the flow separates from the roof and causes the drag to increase.

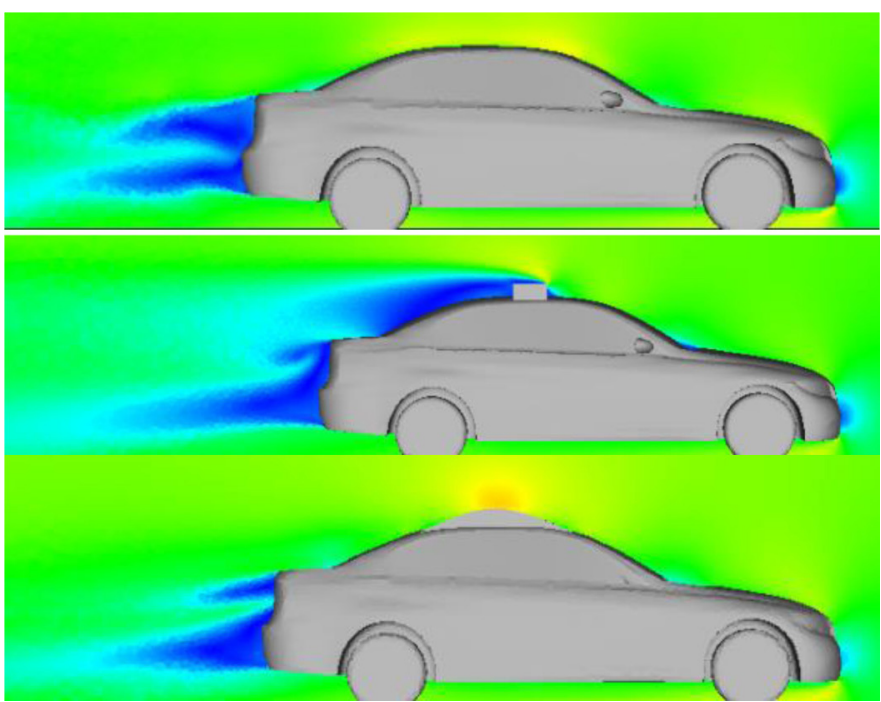

Figure 16. Contours of velocity for original (top), with light bar (middle) and optimum design (bottom) 
As it can be seen in the figure 16 for the optimum case, the low velocity wake (blue color) on the roof disappears and since the flow is more directed towards the ground with the new roof configuration, there is a reduction in the size of the low pressure separation area at the rear of the vehicle. That is why the drag force for the new configuration (Optimum case) is almost as small as the base car.

Modifying the roof shape, through the Bezier curves, will decrease the pressure in front of the light bar and also completely removes the area of separation and low pressure after the light bars, which are mainly responsible for the extra drag production. By implementing the Bezier curve design, as it can be seen in the figure 15 and $\underline{16}$, the flow becomes streamlined and the area of low pressure on the back of the light bar and also rear of the vehicle are eliminated. Eliminating the area of low pressure caused by separation, on the top and rear of the vehicle and well as removing the circulation on the rear, would decrease the drag force.

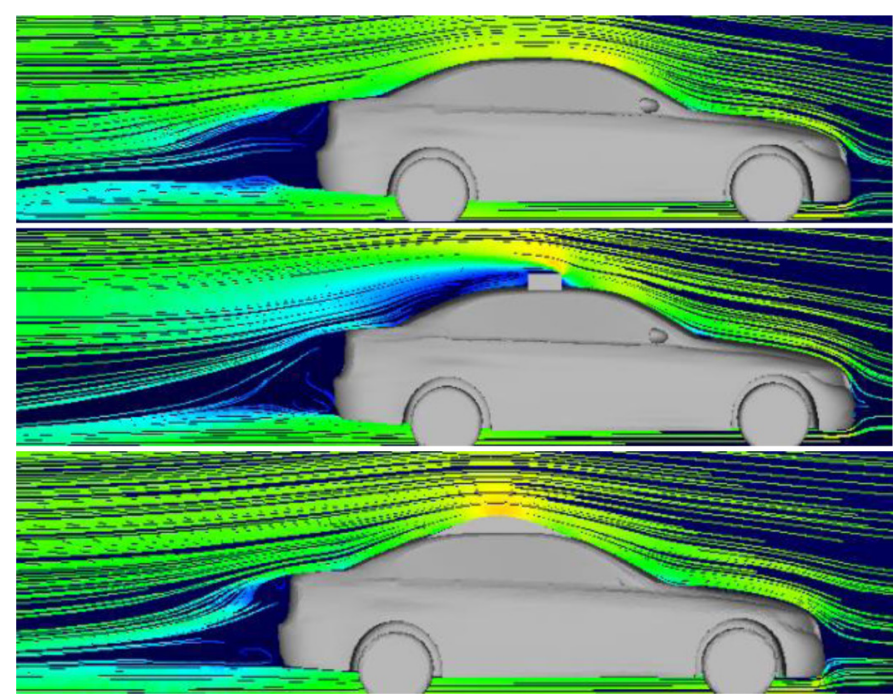

Figure 17. Velocity streamlines for original (top), with light bar (middle) and optimum design (bottom)

The common feature of the optimal configurations is that they eliminate the area of the low pressure after the light bar and reduces the region of flow recirculation at the rear of the vehicle by directing the flow downwards. This optimized design reduces the drag by $30 \%$ compared to the original design with light bar. Note that the restrictions on the design variables, to take account of practical constraints on the size of police car, limits the scope for achieving greater drag reductions. For example, reducing the vehicle height (and/or decreasing its width) at its rear also offers significant drag reducing potential []]

\section{CONCLUSIONS}

Rising fuel costs, coupled with the need to reduce the environmental impact of its fleet operations, are now stimulating interest in improving the aerodynamic design of police cars. This paper is the first to combine CFD with formal optimization methods to investigate the potential for reducing the aerodynamic design of police cars. It has shown that, improving the aerodynamic design of the roof and light-bars in police car conversions can potentially reduce the aerodynamic drag by up to $30 \%$. This would also offer a significant opportunity for reducing the fuel consumption resulting from police fleet operations [11] .

A CFD optimization methodology has been developed and applied to a realistic model of a police car inside the wind tunnel, for minimizing the drag force. High fidelity flow analysis has been combined with a formal optimization framework in order to optimize the shape of the police car with light bar. The roof with light bar design is parameterized into two key design variables and a number of designs are produced using an Optimal Latin Hypercube design of experiments. The choice of objective function and design parametrization has an important influence on the efficiency and effectiveness of the optimization achieved.

Further improvements in fuel consumption could be obtained by optimizing other parts of the vehicle, such as the lower surface and under body features, and taking a multidisciplinary approach to the problem where weight reduction, as is common in the aerospace industry, and potentially crashworthiness are considered. Work is still ongoing and is focusing on developing other parameterization methods in order to achieve further drag reduction.

\section{REFERENCES}

1. Bayraktar, I. and Bayraktar T., Guidelines for CFD Simulations of Ground Vehicle Aerodynamics. 2006.

2. Muyl, F., Dumas L., and Herbert V., Hybrid method for aerodynamic shape optimization in automotive industry. Computers and Fluids, 2004. 33(5): p. 849-858.

3. Katz, J., Aerodynamics of Race Cars. Annual Review of Fluid Mechanics, 2006. 38: p. 27-64.

4. McCallen, R.C., Fred Browand, F., and Ross, J, The Aerodynamics of Heavy Vehicles: Trucks, Buses, and Trains. 19. 2004a: Springer.

5. McCallen, R.C., Salari, K., Ortega, J.M., DeChant, L.J., Hassan, B., Roy, C.J., Pointer W.D., Browand, F., Hammache, M., Hsu, T-Y., Leonard, A., Rubel, M., Chatalain, P., Englar, R., Ross, J., Satram, D., Heineck, J.T., Walker, S., Yaste, D. and Storms, B., DOE's Effort to Reduce Truck Aerodynamic Drag,Joint Experiments and computations Lead to Smart Design. AIAA Fluid Dynamics Conference and Exhibit, 2004b. 34.

6. Raveendran, A., et al., Exterior Styling of an Intercity Transport Bus for Improved Aerodynamic Performance. 2009.

7. Mirables Buil, R.a.C.H., L, Aerodynamic analysis of a vehicle tanker. 2009: J. Fluids Eng.

8. Gilkeson, C.A., et al., An experimental and computational study of the aerodynamic and passive ventilation characteristics of small livestock trailers. Journal of Wind Engineering and Industrial Aerodynamics, 2009. 97(9-10): p. 415-425.

9. Williams, N.A., Gordis J.H., and Letherwood M., Drag Optimization of Light Trucks Using Computational Fluid Dynamics. 2004.

10. Taherkhani, A.R., Computational fluid dynamics based optimisation of emergency response vehicles. 2015, University of Leeds.

11. Thompson, H., Aerodynamic Drag Reduction of Emergency Response Vehicles. Advances in Automobile Engineering, 2015. 2015.

12. N. Bizzarrini F.G., Coiro D.P., Genetic algorithm in wind turbine airfoil design. EWEA 2011, brussels, Belgium, 2011.

13. Khatir, Z., et al., Energy thermal management in commercial breadbaking using a multi-objective optimisation framework. Applied Thermal Engineering, 2015. 80: p. 141-149.

14. Samareh, J.A., Survey of Shape Parameterization Techniques for HighFidelity Multidisciplinary Shape Optimization. AIAA Journal, 2001. 39(5): p. 877-884.

15. Karpowitz, D., Bezier curve fitting method for existing turbine blade design. Journal of applied Engieneering Mathematics, 2005. 3.

16. Giannakoglou, K.C., A Design Method For Turbine Blades Using Genetic Algorithms On Parallel Computers. ECCOMAS 98, 1998. 
17. Pal, S., Biswas P.K., and Abraham A.. Face recognition using interpolated Bezier curve based representation. in Information Technology: Coding and Computing, 2004. Proceedings. ITCC 2004. International Conference on. 2004.

18. Versteeg, H.K. and Malalasekera W., An Introduction to Computational Fluid Dynamics: The Finite Volume Method. 2007: Pearson Education Limited.

19. Bayraktar, I.a.B., T, Guidelines for CFD Simulations of Ground Vehicle Aerodynamics. Commercial Vehicle Engineering Congress and Exhibition,Chicago, Illinois, 2006.

20. Feng, Y., et al., Study on Influence of Mesh Parameters on Vehicle Aerodynamic Drag Coefficient. 2008.

21. http://ecomodder.com/wiki/index.php/Vehicle Coefficient of Drag List. 2014 [cited 2014 23/09/2014].

22. The Road Vehicles Lighting Regulations 1989 (1 November 1989). 2016 [cited 2016 20/007/2016]; Available from: http://www.ukemergency. co.uk/blue-light-use/.

23. Ansys_13.0. http://www.ansys.com/Products/Workflow+Technology/ ANSYS+Workbench+Platform [cited 2012 03/05/2012]; Ansys 13.0].

24. Rao, S.S., Engineering Optimization - Theory and Practice (4th Edition). John Wiley \& Sons.

25. Deb, K., Multi-Objective Optimization Using Evolutionary Algorithms. 2001, New York: John Wiley \& Sons.

26. Michael Wettera, J.W., A comparison of deterministic and probabilistic optimization algorithms for nonsmooth simulationbased optimization Program. Building and Environment. 2004.
27. Wang, S. and Jin X., Model-based optimal control of VAV airconditioning system using genetic algorithm. Building and Environment, 2000. 35(6): p. 471-487.

28. Wright, J.A., Loosemore H.A., and Farmani R., Optimization of building thermal design and control by multi-criterion genetic algorithm. Energy and Buildings, 2002. 34(9): p. 959-972.

29. Murai, M., Sakamoto Y., and Shinozaki T.. An optimizing control for district heating and cooling plant. in Control Applications, 1999. Proceedings of the 1999 IEEE International Conference on. 1999.

30. Magnier, L. and Haghighat F., Multiobjective optimization of building design using TRNSYS simulations, genetic algorithm, and Artificial Neural Network. Building and Environment. 45(3): p. 739-746.

31. Queipo, N.V., et al., Surrogate-based analysis and optimization. Progress in Aerospace Sciences, 2005. 41(1): p. 1-28.

32. Toropov, V.V., Bates S.J., and Querin O.M.. Generation of Extended Uniform Latin Hypercube Designs of Experiments. in Proceedings of the Ninth International Conference on the Application of Artificial Intelligence to Civil, Structural and Environmental Engineering. 2007. Civil-Comp Press, Stirlingshire, UK.

33. Khan, M.A., Noakes C., and Toropov V., Development of a numerical optimization approach to ventilation system design to control airborne contaminant dispersion and occupant comfort. Building Simulation, 2012. 5(1): p. 39-50.

34. Patankar, S.V., Numerical Heat Transfer and Fluid Flow. 1980, New york: McGraw-Hill.

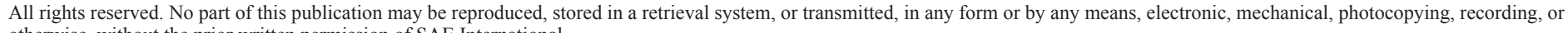
otherwise, without the prior written permission of SAE International. 